\title{
NSV 1907 - a New Eclipsing, Nova-Like Cataclysmic Variable
}

\author{
Stefan Hümmerich ${ }^{\mathrm{a}, \mathrm{b}}$, Rainer Gröbel ${ }^{\mathrm{a}}$, Franz-Josef Hambsch ${ }^{\mathrm{a}, \mathrm{b}, \mathrm{c}}$, Franky Dubois ${ }^{\mathrm{c}, \mathrm{d}}$, Richard Ashley ${ }^{\mathrm{e}}$, Boris T. \\ Gänsicke ${ }^{\mathrm{e}}$, Siegfried Vanaverbeke ${ }^{\mathrm{c}, \mathrm{d}, \mathrm{f}}$, Klaus Bernhard ${ }^{\mathrm{a}, \mathrm{b}}$, Patrick Wils ${ }^{\mathrm{c}}$ \\ ${ }^{a}$ Bundesdeutsche Arbeitsgemeinschaft für Veränderliche Sterne e.V. (BAV), Berlin, Germany \\ ${ }^{b}$ American Association of Variable Star Observers (AAVSO), Cambridge, USA \\ ${ }^{c}$ Vereniging Voor Sterrenkunde (VVS), Brugge, Belgium \\ ${ }^{d}$ Astrolab IRIS, Ieper, Belgium \\ ${ }^{e}$ Department of Physics, University of Warwick, Coventry, UK \\ ${ }^{f}$ Center for Plasma Astrophysics, University of Leuven, Belgium
}

\begin{abstract}
NSV 1907, formerly listed as an irregular variable in variability catalogues, was classified as an Algol-type eclipsing binary in the Catalina Surveys Periodic Variable Star Catalogue. We have identified NSV 1907 as an ultraviolet (UV) bright source using measurements from the GALEX space telescope and detected obvious out-of-eclipse variability in archival photometric data from the Catalina Sky Survey, which instigated a closer examination of the object. A spectrum and extensive multicolour photometric observations were acquired, from which we deduce that NSV 1907 is a deeply eclipsing, nova-like cataclysmic variable. Apart from the orbital variations (deep eclipses with a period of $P \approx 6.63$ hours), changes in mean brightness and irregular short-term variability (flickering) were observed. The presence of a secondary minimum at phase $\varphi \approx 0.5$ was established, which indicates a significant contribution of the companion star to the optical flux of the system. We find possible evidence for sinusoidal variations with a period of $P \approx 4.2 \mathrm{~d}$, which we interpret as the nodal precession period of the accretion disc. No outbursts or VY Scl-like drops in brightness were detected either by the CSS or during our photometric monitoring. Because of its spectral characteristics and the observed variability pattern, we propose NSV 1907 as a new moderately bright long-period SW Sextantis star. Further photometric and spectroscopic observations are encouraged.
\end{abstract}

Keywords: binaries: eclipsing, stars: variables: cataclysmic variables, stars: variables: SW Sex stars, stars: individual: NSV 1907

\section{Introduction}

Cataclysmic variables (CVs) are interacting binary systems which comprise a low-mass secondary star (mostly a red dwarf) losing material to a white dwarf (WD) primary star. They exhibit complex photometric variability, which is characterised by a variety of phenomena like e.g. eclipses, rapid oscillations, ellipsoidal modulation and abrupt and conspicuous brightenings (i.e. dwarf nova eruptions or nova outbursts). Other tell-tale signs of CVs are the presence of strong emission lines in their optical spectra, their blue colour and, especially in the case of magnetic CVs, their X-ray luminosity, all of which are made good use of in searches for this kind of variable stars. For a general review of $\mathrm{CVs}$, the reader is referred to Warner (1995) and Hellier (2001).

Nova-like cataclysmic variables are characterised by high mass transfer rates and prominent steady state accretion discs and do not show large amplitude outbursts. Among this subgroup of CVs, the SW Sextantis stars are set apart by several common traits, which include unusual V-shaped eclipse profiles, single-peaked emission lines exhibiting central absorption dips around orbital phases $\varphi \approx 0.4-0.7$, a substantial orbital phase lag $(0.1-0.2$ cycle) of the ra- dial velocities of the Balmer lines and high-velocity emission $\mathrm{S}$-waves with maximum blueshift near phase $\varphi \approx 0.5$ (Thorstensen et al. 1991; Rodríguez-Gil et al. 2007a). SW Sex systems are not rare but dominate in the period range $2.8 \leq P_{\text {orb }} \leq 4$ hours (Rodríguez-Gil et al. 2007b; Schmidtobreick et al. 2012), just above the period gap. Recently, evidence has been mounting that the SW Sex phenomenon is an evolutionary stage in the life of CVs (Schmidtobreick et al. 2012).

Optical transient surveys such as the Catalina Realtime Transient Survey (CRTS; Drake et al. 2009) and the All-Sky Automated Survey for SuperNovae (ASAS-SN1; Shappee et al. 2014) are routinely discovering new dwarf novae by the hundreds through detection of their outbursts. Equally, X-ray observatories like e.g. ROSAT (Voges et al. 1991), INTEGRAL (Ubertini et al. 2003) or Swift (Cusumano et al. 2010) have contributed to the discovery of many magnetic CVs.

However, our knowledge of the intrinsic population of the nova-like CVs, which neither show large amplitude outbursts nor the X-ray emission typical of their magnetic brethren, is probably still rather incomplete. Here we outline a new method of identifying nova-like variables, and 
report the identification of NSV 1907 as a candidate moderately bright long-period SW Sex star. The method of identification and observations of our target star are presented in Section 2. Our data analysis is described in Section 3 and we conclude in Section 4.

\section{Method of Identification and Observations}

\subsection{Method of Identification}

As has been pointed out above, nova-like cataclysmic variables are difficult to identify because of the absence of large-scale outbursts that are readily detected by optical transient surveys. However, the optical spectra of novalike stars, including the SW Sex stars, are characterised by very blue continua. It is therefore not surprising that a substantial fraction of this class of variables has been discovered in ultraviolet (UV) excess surveys (RodríguezGil et al. 2007b).

As a starting point in the search for nova-like CVs, we have investigated the extensive sample of variable stars compiled in the Catalina Surveys Periodic Variable Star Catalogue (Drake et al. 2014) using UV photometry from the GALEX (Galaxy Evolution Explorer Space Telescope; Martin et al. 2005) satellite, which has been monitoring the sky in $F U V\left(1344-1786 \AA, \lambda_{\text {eff }}=1538.6 \AA\right)$ and NUV $\left(1771-2831 \AA, \lambda_{\text {eff }}=2315.7 \AA\right)$ simultaneously (Morrissey et al. 2007). To this end, both source catalogues were cross-matched and the resultant list of objects was investigated in $F U V$ vs. $N U V$ color space. The result is shown in Fig. 1.

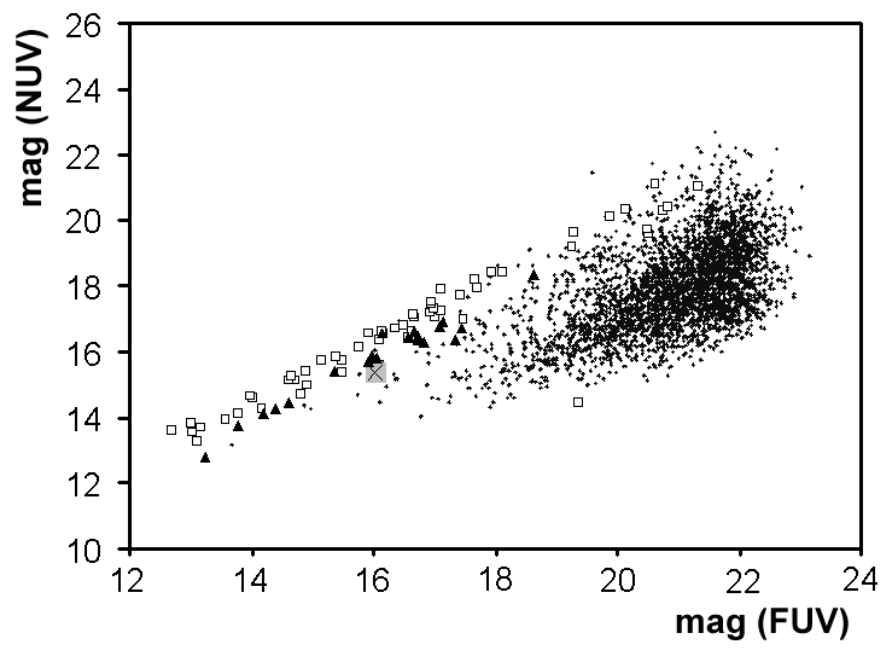

Figure 1: $F U V$ vs. $N U V$ diagram of all sources in the Catalina Surveys Periodic Variable Star Catalogue (Drake et al. 2014) with good GALEX photometry. Post-common envelope binaries are denoted by open squares. Definite or probable SW Sextantis stars from the 'Big List of SW Sextantis Stars' (Hoard et al. 2003) are represented by filled triangles. The grey square marked with a cross indicates the position of NSV 1907.

Groups of interest were identified, like e.g. post-common envelope binaries (class code '16' in Drake et al. 2014; denoted by open squares in Fig. 1) and definite or probable
SW Sex stars from the 'Big List of SW Sextantis Stars' (Hoard et al. 2003) ${ }^{1}$ (represented by filled triangles in Fig. 1). UV bright objects situated near the expected loci of nova-like CVs were investigated in more detail. We concentrated in particular on stars that had been classified as short-period eclipsing binaries in the Catalina Surveys Periodic Variable Star Catalogue, which brought to our attention NSV 1907 ( $F U V=16.028$ mag; $N U V=16.043$ mag; $(F U V-N U V) \approx 0$; position indicated by the grey square marked with a cross in Fig. 1). Inspection of the corresponding light curve from the Catalina Sky Survey (CSS; Drake et al. 2009) resulted in the detection of obvious out-of-eclipse variability, which instigated a closer examination of the object.

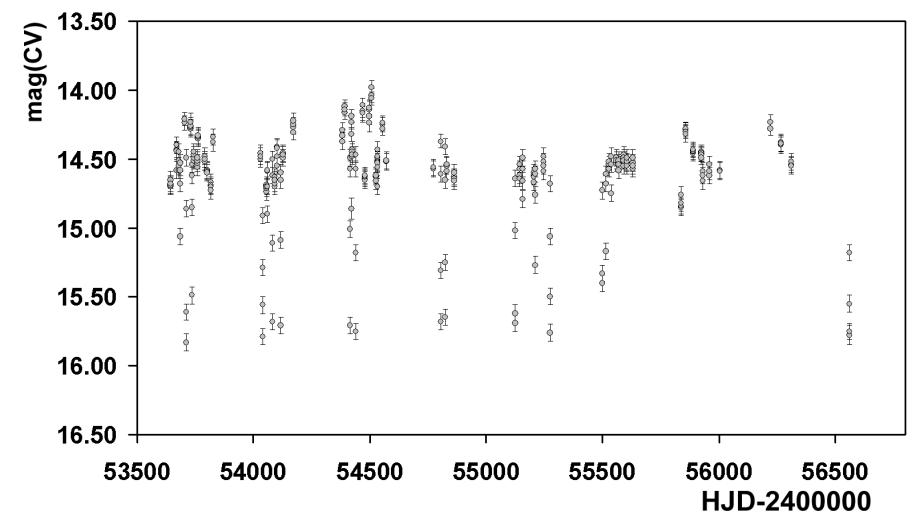

Figure 2: Light curve of NSV 1907, based on data from the Catalina Sky Survey (Drake et al. 2009).

\subsection{Target Star}

The variability of GSC 00104-02193 = USNO-B1.0 09350067535 = 2MASS J05165408+0332525 (RA, Dec (J2000) $=05 \mathrm{~h} 16 \mathrm{~m} 54 \mathrm{~s} .085,+03^{\circ} 32^{\prime} 52^{\prime \prime} .45$; UCAC4 position) was discovered by C. Hoffmeister on Sonneberg plates and the star was announced as a long-period variable with an amplitude of $0.5 \mathrm{mag}$ (Hoffmeister 1963). The star was primarily designated as S 8003 and later included in the New Catalogue of Suspected Variable Stars (Kukarkin \& Kholopov 1982) as NSV 1907. No more information on the type of variability had been available until the object was included in the Catalina Surveys Periodic Variable Star Catalogue (Drake et al. 2014) under the designation of CSS J051654.1+033252 and classified as an Algol-type eclipsing binary (GCVS-type EA) with a period of $P=0.2761060$ $\mathrm{d}$ and an eclipse depth of $1.10 \mathrm{mag}$.

\subsection{Archival Photometry}

Archival photometry of NSV 1907 was procured from the CSS, which observed NSV 1907 during a timespan of about 2915 days. 283 observations of NSV 1907 are available in Data Release 2, which were downloaded from

\footnotetext{
${ }^{1}$ http://www.dwhoard.com/biglist
} 




Figure 3: Phase plot of NSV 1907, based on data from the Catalina Sky Survey (Drake et al. 2009). The data have been folded with a period of $P=0.2761060 \mathrm{~d}$ (Drake et al. 2014). Also included are the CSS light curves of three constant, nearby stars of similar magnitude (denoted as C); see text for details.

the corresponding website ${ }^{2}$. Magnitudes derived from the CSS are unfiltered values that have been calibrated against $V$-band magnitudes and are designated hereafter as 'mag $(C V)^{\prime}$. The CSS light curve is shown in Figure 2. The corresponding phase plot, folded with the ephemeris given in Drake et al. (2014), is given in Fig. 3. Note the obvious out-of-eclipse variability and the irregular depression suggestive of a shallow secondary minimum at phase $\varphi \approx 0.5$. Also included are the CSS light curves of three constant, nearby stars of similar magnitude $(\mathrm{C} 1=\mathrm{CSS}$ J051653.6+033028; C2 = CSS J051648.0+033034; C3 = CSS J051651.3+033311; the light curves of $\mathrm{C} 2$ and C3 have been offset by respectively $-1.0 \mathrm{mag}$ and $-1.8 \mathrm{mag}$ to match the light curve of $\mathrm{C} 1$ ). The constancy of their light curves gives credit to the reality of the observed variability in the CSS light curve of NSV 1907.

\subsection{New CCD Photometry}

To investigate the star in more detail, and to ensure that the observed secondary variability in CSS data is real and not an artefact, we have secured CCD photometric observations of NSV 1907 at three different sites. The instrumentations used are described in the following; an overview of the newly acquired photometry for NSV 1907 is given in Fig. 4.

- Private Observatory, Eckental, Germany In 11 nights from 14/12/2014 to 15/12/2015, extended image series were acquired mostly under uneven sky conditions with a 250/10 SCT in a semiautomated mode and a SBIG ST8XME camera. With

\footnotetext{
${ }^{2}$ http://nesssi.cacr.caltech.edu/DataRelease/
}

120 s exposure time in the $2 \times 2$ binning mode, a total of 1369 measurements were taken (referred to hereafter as the Gröbel dataset). To increase the S/N ratio, no filter was used. Twilight sky-flat images were used for flatfield corrections. The reductions were performed with the MUNIWIN program package (Motl 2011).



Figure 4: Light curve of NSV 1907, based on our own photometric observations, as indicated. The (unfiltered) relative Gröbel data have been shifted by +14.8 mag to match the $V$ observations.

- Remote Observatory Atacama Desert (ROAD), Chile Additional filtered observations were procured at the ROAD observatory (Hambsch 2012), using an Orion Optics, UK Optimized Dall Kirkham 406/6.8 telescope and a FLI 16803 CCD camera. Data were obtained in 20 nights from 07/01/2016 to 19/03/2016. Images were taken through Astrodon Photometric $B, V$, and $I_{\mathrm{C}}$ filters. With 60 s exposure time in the $3 \times 3$ binning mode, a total of 2241 measurements were acquired (referred to hereafter as the ROAD dataset). Twilight sky-flat images were used for flatfield corrections. The reductions were performed with the MAXIM DL program ${ }^{3}$ and the determination of magnitudes using the LesvePhotometry pro$\operatorname{gram}^{4}$.

\footnotetext{
${ }^{3}$ http://www.cyanogen.com

${ }^{4}$ http://www.dppobservatory.net/
} 
- AstroLAB, Ieper, Belgium

Further photometry was acquired at the AstroLAB observatory, Belgium, in 5 nights during the time span $08 / 01 / 2016$ to $13 / 02 / 2016$. The instrumental setup consisted of an 684/4.4 Newton telescope on a Keller alt-azimuth mount and an SBIG STL-6303 E camera. Astrodon $B$ and $V$ filters were used. A total of 591 measurements were taken in the $3 \times 3$ binning mode, with either 60s (dataset 16/01/2016, $\mathrm{V}$ filter; dataset $17 / 01 / 2016, V$ filter) or 120 s (dataset 08/01/2016, $V$ filter; dataset 02/02/2016, $B$ filter) exposure time. The LesvePhotometry program was employed for the determination of magnitudes.

\subsection{Spectroscopic Observations}

A spectrum of NSV 1907 was obtained on the night beginning on the 19th of January 2016 using the Intermediate Dispersion Spectrograph at the $2.5 \mathrm{~m}$ Isaac Newton Telescope (INT) at the Roque de los Muchachos Observatory in La Palma, Canary Islands. The grating used was the $\mathrm{R} 400 \mathrm{~V}$ grating, a medium dispersion grating with a dispersion of $1.55 \AA$ per pixel. The central wavelength was $5513.6 \AA$.

The spectrum is shown in Figure 5. It is the result of combining 3 individual exposures of 400s taken in succession. Since no suitable standard was available in that night's data, the spectrum was flux-calibrated using a standard, SP0946+139 (aka HD 84937), taken with the same instrument setup (grating, slit width and central wavelength) on the night of the 26th January 2016. The spectrum was reduced using the 'molly' reduction software ${ }^{5}$ written by Tom Marsh at the University of Warwick.

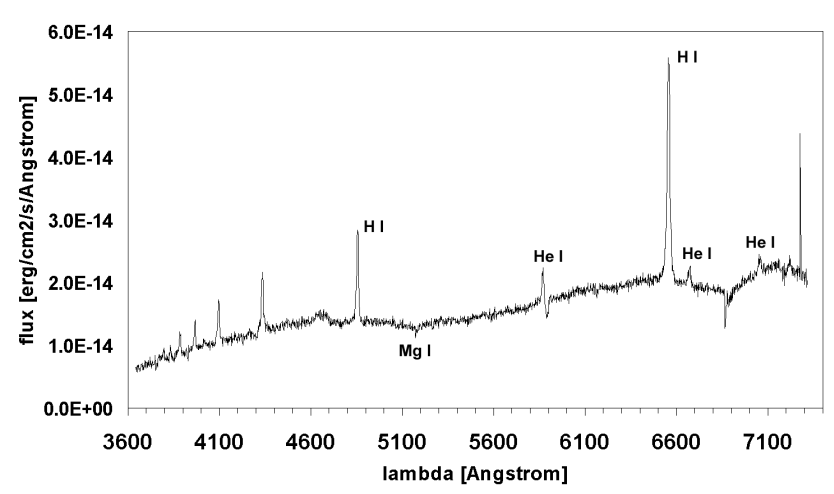

Figure 5: Spectrum of NSV 1907 obtained with the Isaac Newton Telescope (INT). See text for details.

The spectrum (Fig. 5) is typical for a cataclysmic variable, exhibiting the characteristic hydrogen and neutral helium emission lines. In addition to that, there is some evidence of higher excitation lines ('Bowen blend' at around $4640 \AA$, caused by ionised carbon and nitrogen, with possibly some contribution from He II emission at $4686 \AA$ ),

\footnotetext{
${ }^{5}$ http://deneb.astro.warwick.ac.uk/phsaap/software/
}

which indicates the presence of a source of ionising photons (likely an accreting white dwarf). Except for the $\mathrm{H} \alpha$ line, which shows some slight indication of asymmetry, the Balmer emission lines are symmetric and single-peaked, which is reminiscent of SW Sex systems (Thorstensen et al. 1991) and argues against the presence of a high-inclination accretion disc. However, spectroscopic observations with higher resolution are needed to investigate this matter in detail.

The spectrum clearly exhibits secondary star features (Mg I 5168, 5174, $5185 \AA$ ), which goes along well with the observed secondary eclipse (cf. section 3.2 ). This suggests a $\mathrm{K}$ spectral type for the secondary component, which would be expected for the observed long orbital period (Peters \& Thorstensen 2005; cf. also section 3.1). The Na D lines (5892, $5898 \AA$ ) likely show an absorption component from the interstellar medium.

\subsection{Spectral Energy Distribution}

The spectral energy distribution of NSV 1907 is shown in Figure 6, which has been based on data obtained with the VizieR Photometry viewer ${ }^{6}$. Both reddened (filled symbols) and unreddened (open symbols) values are shown. Values in the WISE W3 and W4 bands denote upper limits and are represented by triangles. Reddening estimates have been based on the calculations of Schlafly \& Finkbeiner (2011) which should be considered as the maximum column density; extinction coefficients for the GALEX passbands were taken from Yuan et al. (2013). We estimate an interstellar extinction of $A_{\mathrm{V}} \approx 0.27 \mathrm{mag}$ and $E(B-V) \approx 0.09 \mathrm{mag}$ in the line-of-sight to our target.

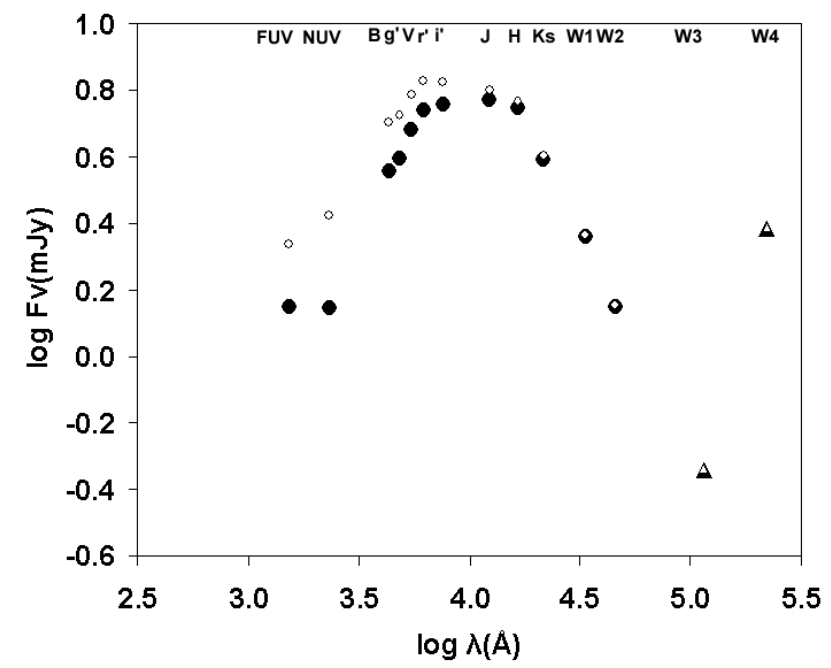

Figure 6: Spectral energy distribution of NSV 1907, based on data obtained with the VizieR Photometry viewer. Filled symbols denote unreddened values, open symbols are based on extinction corrected values. Values in the WISE W3 and W4 bands denote upper limits and are represented by triangles. See text for details.

\footnotetext{
${ }^{6}$ http://vizier.u-strasbg.fr/vizier/sed/
} 
The shape of the SED is typical of nova-like variables, with the accreting WD contributing strongly to the short wavelength region. The secondary star contributes significantly at optical wavelengths, which is in agreement with its presumed spectral type and the observed secondary eclipse at an orbital phase of $\varphi \approx 0.5$.

\section{Data Analysis and Discussion}

\subsection{Period Analysis}

From our newly acquired observations and an analysis of CSS data, the following ephemeris could be derived, where phase zero corresponds to the primary eclipse.

$$
\operatorname{Min}(H J D)=2457060.3785(2)+0.2761069(2) \times E
$$

The corresponding times of minima are given in Table 1. The CSS light curve is not well sampled around the times of primary eclipse and the resulting errors in the times of minima are large; we have thus chosen to exclude CSS data from the period analysis.

Table 1: Observed times of minima.

\begin{tabular}{ccccc}
\hline \hline Observer & HJD-2450000 & Error & Epoch & (O-C) \\
\hline \hline Gröbel & 7060.3790 & 0.00028 & 0 & 0.0005 \\
\hline Gröbel & 7068.3850 & 0.00028 & 29 & -0.0006 \\
\hline Gröbel & 7070.3185 & 0.00022 & 36 & 0.0001 \\
\hline Gröbel & 7080.2582 & 0.00014 & 72 & 0.0000 \\
\hline Gröbel & 7364.6485 & 0.00052 & 1102 & 0.0002 \\
\hline Gröbel & 7387.5648 & 0.00036 & 1185 & -0.0004 \\
\hline Dubois & 7396.4006 & 0.00018 & 1217 & 0.0000 \\
\hline Hambsch & 7401.6470 & 0.00034 & 1236 & 0.0004 \\
\hline Hambsch & 7403.5791 & 0.00036 & 1243 & -0.0003 \\
\hline Gröbel & 7425.3916 & 0.00018 & 1322 & -0.0002 \\
\hline Hambsch & 7425.6677 & 0.00022 & 1323 & -0.0002 \\
\hline Dubois & 7431.4666 & 0.00040 & 1344 & 0.0004 \\
\hline \hline
\end{tabular}

\subsection{Additional Variability and Amplitudes}

From our new observations, the reality of the additional variability seen in CSS data in addition to the eclipses (short-term irregular variability, long-term variations) could be established, as well as the presence of a secondary minimum which seems to be a constant feature and takes the form of a small, irregular depression at phase $\varphi \approx 0.5$. The primary minimum seems to be slightly variable, too. Figure 7, which has been based on part of the unfiltered Gröbel data, nicely illustrates the observed additional variability. Detailed views of primary eclipses are presented in Figure 8.

During our photometric coverage, the observed changes in mean brightness of the system outside of primary eclipse amounted to $\sim 0.3 \mathrm{mag}(C R), \sim 0.5 \mathrm{mag}(V)$, and $\sim$



Figure 7: Phase plot of NSV 1907, based on part of the unfiltered Gröbel data (as indicated in the inset legend) and folded with the ephemeris given in Eq. (1). Note the obvious out-of-eclipse variability and the presence of a secondary minimum at phase $\varphi \approx 0.5$.

$0.5 \mathrm{mag}(B)$, respectively. Archival data from the CSS indicate mean brightness variations of up to $0.7 \mathrm{mag}(\mathrm{CV})$, which becomes especially obvious during the active phase around HJD 2454500.
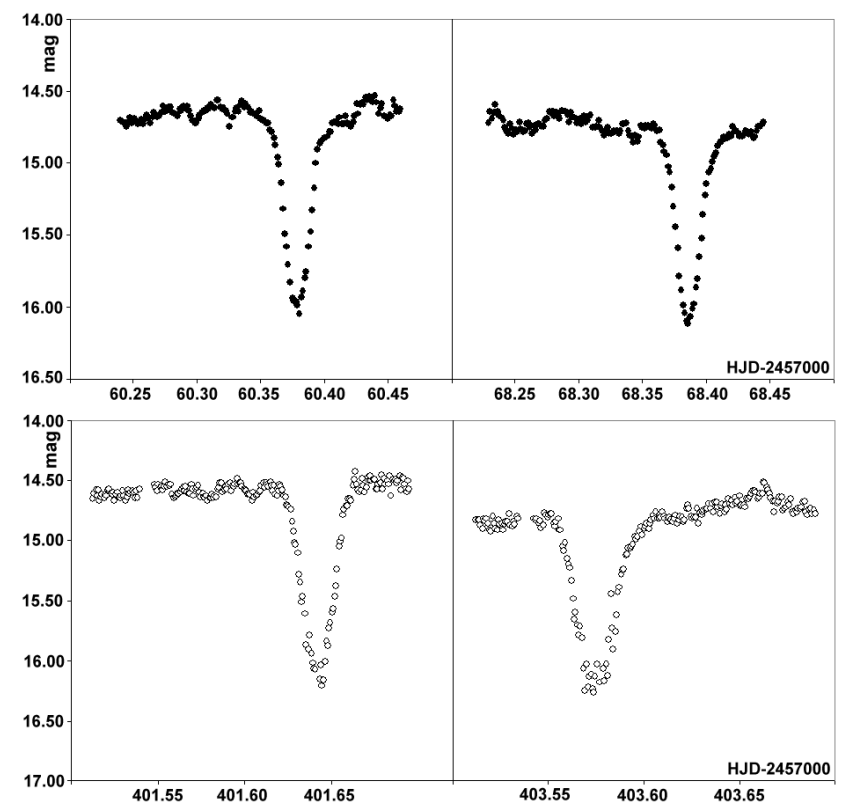

Figure 8: Detailed view of eclipses of NSV 1907. The plots have been based on unfiltered Gröbel data (upper panels; full circles) and ROAD $V$ data (lower panels; open circles). Note the short-term irregular variability that is present outside of eclipses.

Unfortunately, no simultaneous multicolour coverage of the primary eclipse exists. However, judging from the long-term light curve, the primary eclipse is a rather stable feature. We have therefore derived approximate eclipse amplitudes employing the best-covered eclipses in $B$ (around 
HJD 2457431.46), $V$ (around 2457422.63), and $I_{\mathrm{C}}$ (around HJD 2457425.67). An overview of these eclipses is presented in Fig. 9. Although the scatter in the $B$ and $I_{\mathrm{C}}$ observations is relatively large, it is obvious that the amplitude of the eclipse increases to shorter wavelengths $\left(\Delta B>\Delta V>\Delta I_{\mathrm{C}}\right)$, indicating that the hot object is eclipsed around phase $\varphi \approx 0$. We derive eclipse amplitudes of $\Delta B \approx 2.0 \mathrm{mag}, \Delta V \approx 1.5 \mathrm{mag}$, and $\Delta I_{\mathrm{C}} \approx 0.8$ mag.

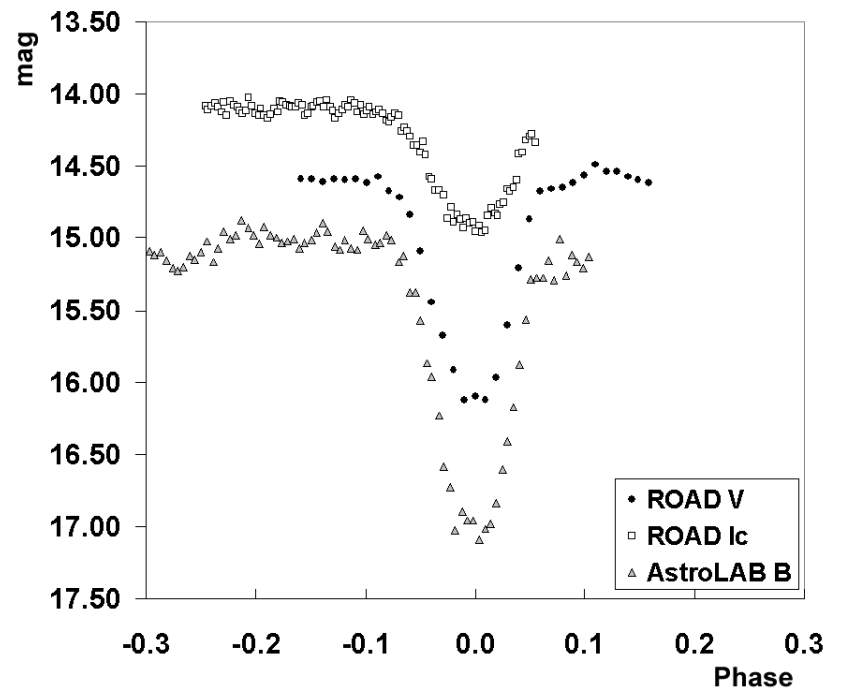

Figure 9: Detailed view of eclipses based on AstroLAB $B$ and ROAD $V I_{\mathrm{C}}$ data. Outlying datapoints have been removed by visual inspection. The data were acquired on different orbital cycles (see text for details).

The presence of a secondary minimum in the light curve of NSV 1907 is remarkable as this is seldom observed in eclipsing cataclysmic variables. There are exceptions like e.g. the SW Sextantis system V363 Aur (Thoroughgood et al. 2004). This star exhibits a secondary eclipse in the $R$ band light curve, which is absent in the $B$ band, implying that this feature is stronger at longer wavelengths. Apparently, the reverse holds true for NSV 1907; from Fig. 10, it becomes apparent that the amplitude of the secondary eclipse is greater in $B$ than in $V$.

Likely, then, different mechanisms are at work in NSV 1907. It seems probable that the accretion disc occults the companion star that contributes a significant amount to the optical flux output of the system, which results in the observed secondary eclipse at phase $\varphi \approx 0.5$. The reflection effect (heating of the companion star by the WD primary and/or the accretion disc) may be important in this respect. There could also be material above and below the disc plane that contributes to the eclipse of the secondary.

\subsection{The 4.2 day period - the nodal precession period?}

We have searched for periodic signals other than the orbital period in the ROAD $V$ dataset, which covers the most consecutive orbital cycles. To this end, eclipses were

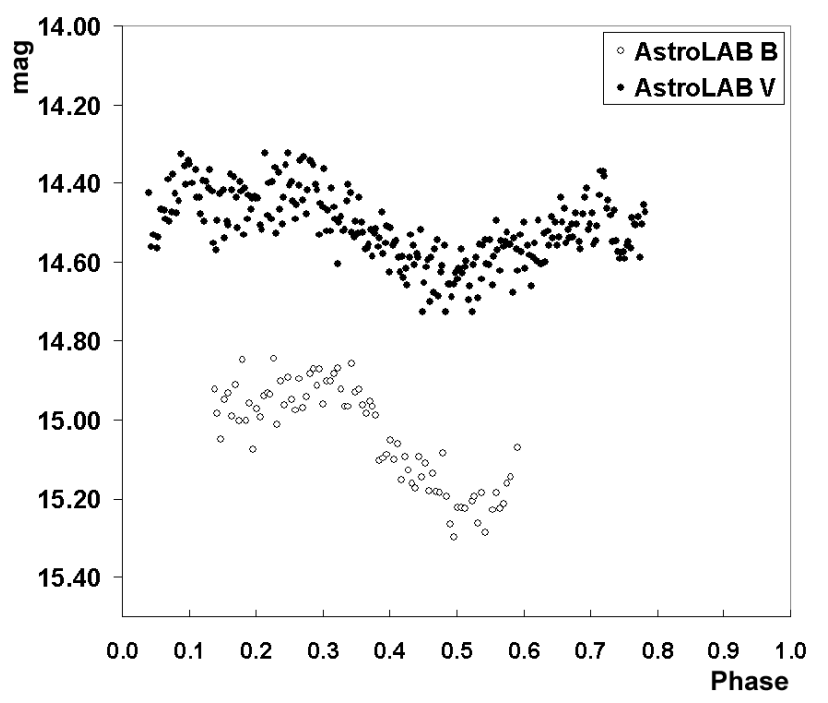

Figure 10: Detailed view of secondary eclipses based on AstroLAB $B V$ data. Outlying datapoints have been removed by visual inspection. The data were acquired on different orbital cycles (see text for details).

removed by visual inspection and the resulting data were searched in the frequency range of $0<f(c / d)<3$ using PERIOd04 (Lenz \& Breger 2005). We find possible evidence for sinusoidal variations with a period of $P \approx 4.2 \mathrm{~d}$ (Fig. 11), which is reminiscent of the findings of de Miguel et al. (2016) who find a period of $P \approx 3.68 \mathrm{~d}$ from an analysis of long-term photometric observations on the nova-like variable UX UMa. The authors interpret this signal as the signature of a retrograde precession of the accretion disc.

If we adopt the same interpretation for NSV 1907, i.e. that the 4.2 day period is the nodal precession period, the superhump period $P_{\text {sh }}$ can be deduced from $f_{\text {sh }}=f_{\text {orb }}$ $+f_{\text {nodal }}$. Assuming $P_{\text {orb }}=0.2761 \mathrm{~d}\left(f_{\text {orb }}=3.6219 \mathrm{c} / \mathrm{d}\right)$ and $P_{\text {nodal }}=4.211 \mathrm{~d}\left(f_{\text {nodal }}=0.2375 \mathrm{c} / \mathrm{d}\right)$, a superhump period of $P_{\mathrm{sh}}=0.2591 \mathrm{~d}\left(f_{\mathrm{sh}}=3.8594 \mathrm{c} / \mathrm{d}\right)$ is derived. From this, the observed fractional period excess of the superhump period $\epsilon=\left(P_{\text {sh }}-P_{\text {orb }}\right) / P_{\text {orb }}$ (Patterson et al. 2005) can be derived, which - in the case of NSV 1907 amounts to $\epsilon \approx 0.06$.

It has been shown that $\epsilon$ is strictly a function of the mass ratio $q=M_{2} / M_{1}$ (Patterson et al. 2005). Using equation (8) of Patterson et al. (2005), we derive $q \approx 0.25$. Considering the long period of NSV 1907, which allows for a bigger and more massive secondary star than found in most other nova-like variables, this value seems reasonable (cf. Fig 9 of Patterson et al. 2005).

However, because of the short time baseline of our data, these values have to be regarded as very preliminary only. More observations are needed to confirm the reality of the proposed period and verify its consistency over a longer timespan. Monitoring of the secondary eclipse might also be useful in establishing the presence of a tilted accretion disc in the system, in which case the secondary eclipse should be variable in time as a result of the changing pro- 

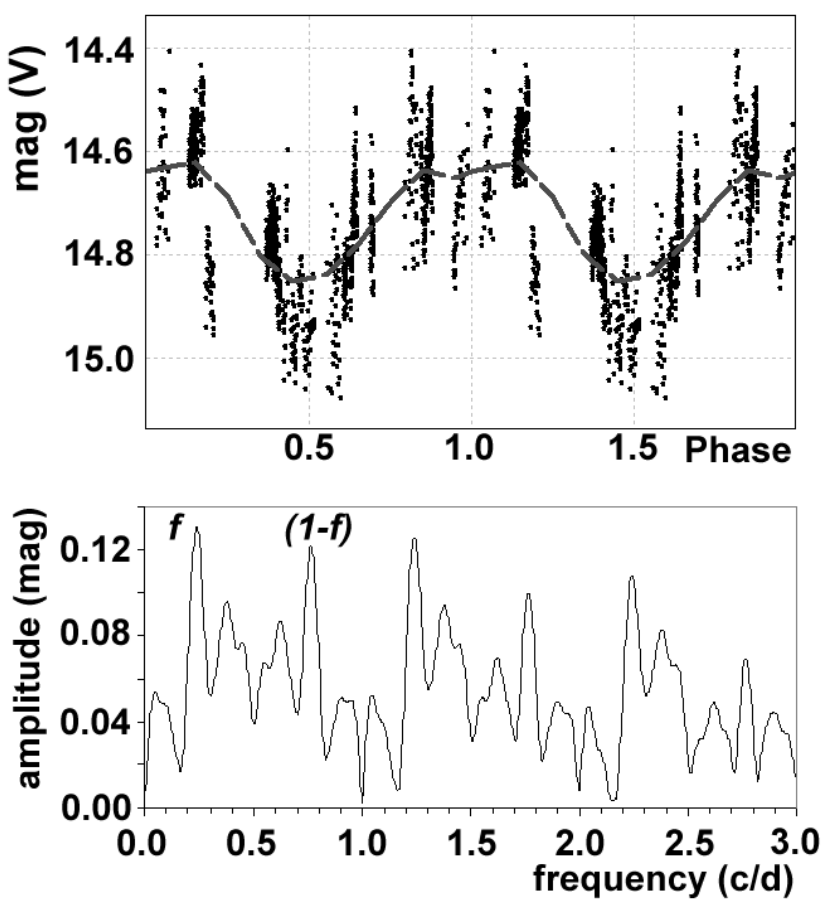

Figure 11: ROAD $V$ data, folded on the proposed 4.2 day $(f=$ $0.2375 \mathrm{c} / \mathrm{d}$ ) variations (upper panel; eclipses have been removed by visual inspection) and the corresponding power spectrum (lower panel), which is dominated by $f,(1-f)$, and the corresponding daily aliases.

jected area of the disc and WD system.

\section{Conclusion}

From our observations, we deduce that NSV 1907 is a deeply eclipsing, nova-like cataclysmic variable. Apart from the orbital variations (eclipses), changes in mean brightness and irregular short-term variability (flickering) were observed. The presence of a secondary minimum at phase $\varphi \approx 0.5$ was established. This is rarely observed in CVs and indicates that the contribution of the companion star is not entirely negligible as compared to the luminosity of the hot object. We have searched for periodic variability other than the orbital period and find possible evidence for sinusoidal variations with a period of $P \approx 4.2 \mathrm{~d}$, which we interpret as the nodal precession period. However, more observations are needed to confirm or disprove the reality of our finding.

No outbursts or VY Scl-like drops in brightness were observed either by the CSS or during our photometric monitoring. Because of its spectral characteristics (singlepeaked emission lines, high-excitation lines) and the observed variability pattern (high-inclination, eclipsing system; V-shaped eclipses; mean brightness changes; flickering), we propose NSV 1907 as a new member of the SW Sextantis class.

In fact, the star's properties and light curve are strongly reminiscent of other long-period SW Sex systems like e.g.
V363 Aur (the longest-period definite SW Sex star with $P_{\text {orb }} \approx 7.71 \mathrm{~h}$ ), which, incidentally, also exhibits a conspicuous secondary eclipse and secondary star features in its spectrum (Thoroughgood et al. 2004). Figure 12 shows the orbital period distribution of SW Sex stars. The plot has been based on data from the 'Big List of SW Sextantis Stars' (Hoard et al. 2003). Only definite members of the group and probable candidates have been considered. With an orbital period of $P \approx 6.63$ hours, NSV 1907 - if confirmed as a member - would be the SW Sex star with the fourth longest period.

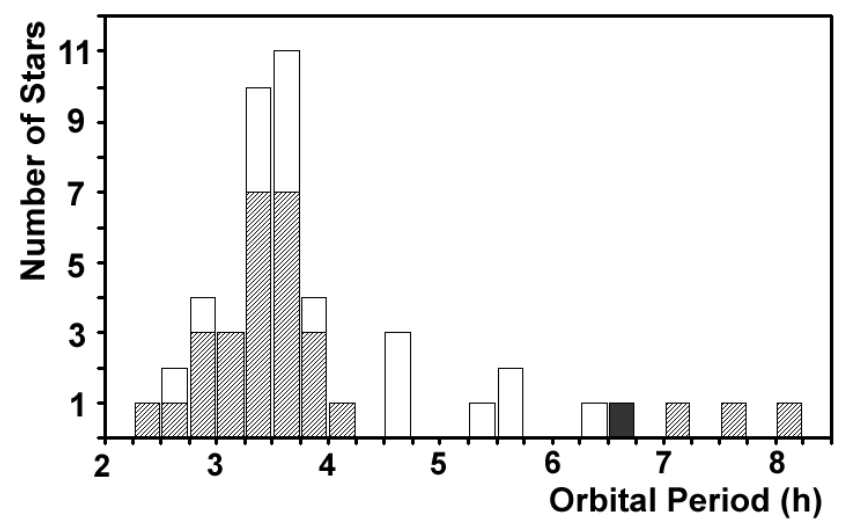

Figure 12: Orbital period distribution of SW Sex stars, based on data from 'The Big List of SW Sextantis Stars' (Hoard et al. 2003). Only definite SW Sex stars (dashed area) and probable candidates (white area) have been considered. The position of NSV 1907 is indicated by the dark area.

Further photometric and spectroscopic observations are encouraged. Time-resolved spectroscopy, in particular, should prove valuable in investigating class-defining features such as the radial velocity variations of the emission lines, which exhibit maximum blueshift near an orbital phase of $\varphi_{\text {orb }} \approx$ 0.5 , or the typical displacement of the radial velocity curve of the line wings (Rodríguez-Gil et al. 2007a).

\section{Acknowledgements}

We thank Tom Marsh at the University of Warwick for the use of the 'molly' reduction software and Marek Skarka for helpful discussions. The research leading to these results has received funding from the European Research Council under the European Union's Seventh Framework Programme (FP/20072013) / ERC Grant Agreement n. 320964 (WDTracer). This research has made use of data from the Catalina Sky Survey. The CSS survey is funded by the National Aeronautics and Space Administration under Grant No. NNG05GF22G issued through the Science Mission Directorate Near-Earth Objects Observations Program. The CRTS survey is supported by the U.S. National Science Foundation under grants AST0909182. Furthermore, use was made of the SIMBAD database and the VizieR catalogue access tool operated at CDS, Strasbourg, France. 


\section{References}

Cusumano, G., La Parola, V., Segreto, A. et al. 2010, A\&A, 524, 64 de Miguel, E., Patterson, J., Cejudo, D. et al. 2016, MNRAS, 457, 1447

Drake, A. J., Djorgovski, S. G., Mahabal, A. et al. 2009, ApJ, 696, 870

Drake, A. J., Graham, M. J., Djorgovski, S. G. et al. 2014, ApJS, 213,9

Hambsch, F.-J. 2012, JAVSO, 40, 1003

Hellier, C. 2001, Cataclysmic Variable Stars, Springer, London

Hoard, D. W., Szkody, P., Froning, C. S. et al. 2003, AJ, 126, 2473

Hoffmeister, C. 1963, Astronomische Nachrichten, 287, 169

Kukarkin, B. V., Kholopov, P. N. 1982, Publication Office Nauka, Moscow, 287, 169

Lenz, P., \& Breger, M. 2005, Communications in Asteroseismology, 146,53

Martin, D. C., Fanson, J., Schiminovich, D. 2005, ApJ, 619, 1

Morrissey, P., Conrow, T., Barlow, T. A. et al. 2007, ApJS, 173, 682

Motl, D. 2011, C-Munipack, http://c-munipack.sourceforge.net

Patterson, J., Kemp, J., Harvey, D. A. 2005, PASP, 117, 1204

Peters, C. S., \& Thorstensen, J. R. 2005, PASP, 117, 1386

Rodríguez-Gil, P., Schmidtobreick, L., Gänsicke, B. T. 2007, MNRAS, 374, 1359

Rodríguez-Gil, P., Gänsicke, B. T., Hagen, H.-J. et al. 2007, MNRAS, 377,1747

Schlafly, E. F., \& Finkbeiner, D. P. 2011, ApJ, 737, 103

Schmidtobreick, L., Rodríguez-Gil, P., Gänsicke, B. T. 2012, MmSAI, 83, 610

Shappee, B. J., Prieto, J. L., Grupe, D. 2014, ApJ, 788, 48

Thoroughgood, T. D., Dhillon, V. S., Watson, C. A. 2004, MNRAS, 353, 1135

Thorstensen, J. R., Davis, M. K., Ringwald, F. A. 1991, AJ, 102, 683

Ubertini, P., Lebrun, F., Di Cocco, G. et al. 2003, A\&A, 411, L131

Voges, W., Aschenbach, B., Boller, Th. 1999, A\&A, 349, 389

Warner, B. 1995, Cataclysmic Variable Stars, Camb. Astrophys. Ser., Vol. 28

Yuan, H. B., Liu, X. W., \& Xiang, M. S. 2013, MNRAS, 430, 2188 